\title{
Modified Asphaltene Capillary Deposition Unit: A Novel Approach to Inhibitor Screening
}

\author{
Saugata Gon, David M. Fouchard
}

\section{Supporting Information}

Capillary cleaning procedure: The new capillary tube (before any crude oil was ever flown through it) was connected to isco pump $\mathrm{C}$ and xylene was flown through it at various flow rates. The pressure head observed at Isco pump $\mathrm{C}$ with the new capillary tube was noted down as a reference point. In-order to clean the capillary unit for reuse after each run, the system was thoroughly cleaned by toluene, iso-propanol and xylene. As toluene wash was the first cleaning cycle, careful visual inspection was carried out for the solvent coming out of the capillary tube. Once transparent solvent started coming out of the system toluene was flown through the system for another hour at $0.5 \mathrm{ml} / \mathrm{min}$ flow rate, followed by iso-propanol wash $(30 \mathrm{~min}$ at $0.5 \mathrm{ml} / \mathrm{min}$ flow rate) and xylene wash (30 $\mathrm{min}$ at $0.5 \mathrm{ml} / \mathrm{min}$ flow rate). At the end of xylene wash the xylene flow rate was varied as per the reference flow rate shown in table S-1, and the isco pressure head was noted. If the Isco pressure head was not higher than the reference pressure head it was assumed that the capillary unit was cleaned thoroughly and the system was ready for the next experiment. Table S-1 shows the reference pressure head and a pressure head obtained for a partially clogged capillary unit. If the capillary unit showed higher pressure head during xylene wash it was thoroughly cleaned again with alternating toluene flow $(0.5 \mathrm{ml} / \mathrm{min}$ flow rate) for $30 \mathrm{~min}$ followed by soaking in toluene for another $30 \mathrm{~min}$. The system was then cleaned with xylene again and the xylene flow rate was 
varied for another comparison against the new capillary pressure head. The process was repeated until we were certain that the unit was thoroughly clean.

\begin{tabular}{|l|l|l|}
\hline \multicolumn{3}{|c|}{ Xylene } \\
\hline $\begin{array}{l}\text { Flow rate, } \\
\mathrm{ml} / \mathrm{min}\end{array}$ & $\begin{array}{l}\text { Isco Pressure } \\
\text { head (new } \\
\text { capillary), psi }\end{array}$ & $\begin{array}{l}\text { Pressure head, } \\
\text { (partially clogged } \\
\text { capillary), psi }\end{array}$ \\
\hline 0.2 & 4 & 5 \\
\hline 1 & 18 & 20 \\
\hline 3 & 53 & 57 \\
\hline 5 & 95 & 99 \\
\hline 8 & 167 & 174 \\
\hline 10 & 222 & 230 \\
\hline 20 & 530 & 551 \\
\hline
\end{tabular}

Table S-1: Comparison of Isco pump C pressure head during xylene wash

\section{Error calculation for understanding statistical significance of deposition rate: A linear}

regression analysis of total deposit collected against experimental run time as discussed in the article suggested that the intercept value could be accounted for residual oil. The residual oil component could be subtracted from the total deposit to calculate asphaltene deposit inside the capillary. Deposition rate for total deposit and asphaltene deposit could be calculated by dividing the respective value by experimental run time (except for the zero time run). Standard deviation could be calculated for total deposition rate value and asphaltene deposition rate values obtained for individual sets of data points as shown in table S-2. The respective error could be calculated by dividing the standard deviation by square root of the respective data points. 


\begin{tabular}{|c|c|c|c|c|c|c|c|c|c|c|}
\hline \multirow[t]{6}{*}{ Blank } & $\begin{array}{l}\text { Run } \\
\text { time, } \\
\mathrm{h}\end{array}$ & $\begin{array}{l}\text { Total } \\
\text { deposi } \\
\text { t, g }\end{array}$ & $\begin{array}{l}\text { Depositi } \\
\text { on rate, } \\
\mathrm{g} / \mathrm{h}\end{array}$ & $\begin{array}{l}\text { Stand } \\
\text { ard } \\
\text { deviat } \\
\text { ion } \\
\end{array}$ & Error1 & $\begin{array}{l}\text { Residual oil } \\
\text { from linear } \\
\text { regression, } \mathrm{g}\end{array}$ & $\begin{array}{l}\text { Total } \\
\text { asphaltene } \\
\text {,g }\end{array}$ & $\begin{array}{l}\text { Depositi } \\
\text { on rate, } \\
\mathrm{g} / \mathrm{h}\end{array}$ & $\begin{array}{l}\text { Standar } \\
\mathrm{d} \\
\text { deviati } \\
\text { on } \\
\end{array}$ & Error2 \\
\hline & 14.47 & 0.499 & 0.034 & \multirow[t]{5}{*}{0.024} & \multirow[t]{5}{*}{0.01} & \multirow[t]{5}{*}{0.210} & 0.290 & 0.020 & \multirow[t]{5}{*}{0.004} & \multirow[t]{5}{*}{0.002} \\
\hline & 8.61 & 0.396 & 0.046 & & & & 0.187 & 0.022 & & \\
\hline & 13.44 & 0.487 & 0.036 & & & & 0.278 & 0.021 & & \\
\hline & 2.78 & 0.243 & 0.087 & & & & 0.033 & 0.012 & & \\
\hline & 3.49 & 0.263 & 0.075 & & & & 0.053 & 0.015 & & \\
\hline \multirow[t]{3}{*}{$\bar{A}$} & 14.98 & 0.608 & 0.041 & \multirow[t]{3}{*}{0.019} & \multirow[t]{3}{*}{0.01} & \multirow[t]{3}{*}{0.223} & 0.385 & 0.026 & \multirow[t]{3}{*}{0.003} & \multirow[t]{3}{*}{0.002} \\
\hline & 4.04 & 0.299 & 0.074 & & & & 0.076 & 0.019 & & \\
\hline & 11.82 & 0.494 & 0.042 & & & & 0.271 & 0.023 & & \\
\hline \multirow[t]{2}{*}{ B } & 17 & 0.599 & 0.035 & \multirow[t]{2}{*}{0.003} & \multirow[t]{2}{*}{0.002} & \multirow[t]{2}{*}{0.255} & 0.344 & 0.020 & \multirow[t]{2}{*}{0.001} & \multirow[t]{2}{*}{0.0006} \\
\hline & 12.42 & 0.492 & 0.040 & & & & 0.238 & 0.019 & & \\
\hline \multirow[t]{2}{*}{$\mathrm{C}$} & 14.06 & 0.472 & 0.034 & \multirow[t]{2}{*}{0.004} & \multirow[t]{2}{*}{0.003} & \multirow[t]{2}{*}{0.269} & 0.203 & 0.014 & \multirow[t]{2}{*}{0.001} & \multirow[t]{2}{*}{0.0006} \\
\hline & 10.46 & 0.408 & 0.039 & & & & 0.139 & 0.013 & & \\
\hline
\end{tabular}

Table S-2: Deposition rate error calculation. A, B and C refer to the data sets for the oil treated with inhibitor A, B and C. Error1 refers to the standard error of deposition rate without oil entrainment correction as referred in Table 1 of the main article. Error 2 refers to the standard error of deposition rate post oil entrainment correction as referred in Table 1 of the main article.

Detailed part list: Information regarding the detailed part list for the modified asphaltene capillary deposition unit used for this study is provided below in Table S-3. 


\begin{tabular}{|c|c|c|c|c|c|c|c|}
\hline Item & Size & $\begin{array}{l}\text { Other } \\
\text { specification }\end{array}$ & Material & $\begin{array}{l}\text { Pressure } \\
\text { rating/range, } \\
\text { psi }\end{array}$ & $\begin{array}{l}\text { Error } \\
\text { Margin }\end{array}$ & Voltage & Vendor \\
\hline Tubing & $1 / 16$ in & & $316 \mathrm{ss}$ & 4500 & & & Swagelok \\
\hline Tubing & $1 / 8$ in & & $316 \mathrm{ss}$ & 4500 & & & Swagelok \\
\hline Capillary coil & $1 / 16$ in & & $316 \mathrm{ss}$ & 4500 & & & Swagelok \\
\hline Valves & $1 / 8$ in at ports & & $316 \mathrm{ss}$ & 2500 & & & Swagelok \\
\hline $\begin{array}{l}\text { Pressure sensor } \\
(\mathrm{P} 1, \mathrm{P} 2, \mathrm{P} 4, \mathrm{P} 5)\end{array}$ & $1 / 4$ in & & $316 \mathrm{ss}$ & $0-5000$ & $.5 \% \mathrm{FS}$ & & AST \\
\hline $\begin{array}{l}\text { Pressure sensor } \\
\text { (P3) }\end{array}$ & $1 / 4$ in & & $316 \mathrm{ss}$ & $0-1000$ & $.25 \% \mathrm{FS}$ & & Omega \\
\hline Transformer & & & 316 ss & 100 & & $\begin{array}{l}110 \mathrm{VAC} \\
\text { to } 24 \\
\text { VDC } \\
\end{array}$ & Frys \\
\hline 1 inlet Manifolds & $1 / 8$ at ports & & $316 \mathrm{ss}$ & 4500 & & & Valco \\
\hline $\begin{array}{l}\text { Temperature } \\
\text { Probes }\end{array}$ & $1 / 8$ in & Type K & $316 \mathrm{ss}$ & & & & Omega \\
\hline $\begin{array}{l}\text { Temperature } \\
\text { Probes }\end{array}$ & $1 / 8$ in & Type J & $316 \mathrm{ss}$ & & & & Omega \\
\hline Pumps & $\begin{array}{l}1 / 8 \text { in ports } \\
\text { with a } 260 \mathrm{ml} \\
\text { accumulator. }\end{array}$ & & & & & & Teledyne \\
\hline Data logger & & & & & & $110 \mathrm{VAC}$ & DataQ \\
\hline Convection Oven & $1.9 \mathrm{CU}$ & $300 \mathrm{C} \max$ & & & & $110 \mathrm{VAC}$ & Binder \\
\hline $\begin{array}{l}\text { Back Pressure } \\
\text { Regulator }\end{array}$ & $1 / 8$ in & & $316 \mathrm{ss}$ & 100 & & & Equilibar \\
\hline Stand & & & Aluminum & & & & In House \\
\hline $\begin{array}{l}\text { Transfer cylinder } \\
\text { (Accumulators) }\end{array}$ & 1 liter & & $316 \mathrm{ss}$ & 800 & & & $\begin{array}{l}\text { McMaster- } \\
\text { Carr }\end{array}$ \\
\hline Pan & 2 liters & & $316 \mathrm{ss}$ & & & & $\begin{array}{l}\text { McMaster- } \\
\text { Carr }\end{array}$ \\
\hline Air compressor & & & & 100 & & $110 \mathrm{VAC}$ & \\
\hline Heater & & 1200 watts & & & & & Jkem \\
\hline Heater bands & & $375 \mathrm{~F}$ max & Silicone & & & & $\begin{array}{l}\text { McMaster- } \\
\text { Carr }\end{array}$ \\
\hline
\end{tabular}

Table S-3: Detailed part list for the asphaltene capillary deposition unit 